\title{
Análisis de las Interacciones entre Alumnos Participando en Proyectos de Diseño Colaborativo Multinacional
}

\author{
Jared Roberto Ocampo Martínez ${ }^{1}$ \\ Roberto Enrique Rodríguez Arriaga \\ UNITEC, San Pedro Sula, Honduras.
}

(Recibido: Diciembre, 2014; Aceptado: Septiembre, 2016)

\begin{abstract}
Resumen:
La globalización y los nuevos modelos de negocios que se han generado como respuesta a este nuevo paradigma requieren de ingenieros que puedan trabajar en equipos multinacionales. Esto hace que sea necesario que los estudiantes desarrollen competencias que les permitan trabajar de manera colaborativa en equipos geográficamente dispersos. El presente artículo busca evaluar las interacciones existentes entre estudiantes participando en el proyecto "América e Italia por el Diseño" y revisar como estas afectan el desarrollo y finalización de un proyecto de diseño de productos. Esta iniciativa reúne estudiantes de seis países diferentes quienes trabajan de forma colaborativa durante un trimestre. Finalmente, esta investigación busca sugerir prácticas que ayuden a que los estudiantes de Unitec que participan en este proyecto tengan una participación más significativa en el mismo.
\end{abstract}

Palabras Claves: Aprendizaje Basado en Proyectos, Diseño de Productos, Geográficamente Disperso, Equipos Virtuales Globales, Colaboración Global Estudiantil.

\begin{abstract}
:
Globalization and new business models that have been generated in response to this new paradigm require engineers who are able to work in multinational teams. This makes it necessary for students to develop skills that enable them to work collaboratively in geographically dispersed teams. This article seeks to evaluate the interactions between students participating in the project "America and Italy by Design" and review how such interactions are affecting the development and completion of a product design project. This initiative brings together students from six different countries who work in collaborative manner during a trimester. Finally, this research aims to suggest practices that may help UNITEC students to be involved in a more significant way in the project.
\end{abstract}

Keywords: Project Based Learning, Product Design, Geographically Dispersed, Global Virtual Teams, Student Global Collaboration.

\section{Introducción}

Una de las dificultades que enfrentan las empresas es la necesidad que tienen de responder de manera más rápida a los vertiginosos cambios de requerimientos de sus clientes. Debido a que la vida útil de los productos ha disminuido dramáticamente, existe una necesidad de

\footnotetext{
${ }^{1}$ Autor para correspondencia. Email: jared.ocampo@unitec.edu
} 
desarrollar nuevos productos de manera más rápida y respondiendo simultáneamente a la creciente demanda de aumentar su calidad y reducir sus costos. Para poder diseñar y manufacturar productos nuevos con las restricciones de tiempo y calidad requeridas en la actualidad, existe la necesidad de habilidades en diferentes campos de manera que esos productos puedan alcanzar las características requeridas por sus clientes. Una de las maneras en que estos problemas pueden ser abordados es a través del uso de desarrollo de productos colaborativo (también conocido como diseño cooperativo, diseño concurrente y diseño interdisciplinario). Este tipo de colaboración permite que diferentes expertos participen en la solución de un problema.

Interesantemente, debido a la globalización, son muchas las compañías que se ven forzadas a pasar por una serie de alianzas estratégicas, adquisiciones y fusiones que obligan a la formación consecuente de equipos de trabajo que se encuentran dispersos globalmente (Zavbi y Tavcar, 2004). Por lo tanto, las empresas no solo necesitan diseñar y fabricar productos que respondan de mejor forma a las necesidades de sus clientes, sino que deben hacerlo mientras hacen frente a los beneficios y desafíos de tener recursos humanos que están organizacionalmente, geográficamente y culturalmente dispersos por el mundo. Para poder enfrentar estos desafíos es conveniente contar con personal (recursos humanos) que tenga experiencia trabajando en este tipo de ambientes, y sepa sacar provecho de sus ventajas y entienda como superar las dificultades que están presentes en este tipo de interacciones.

La universidad y particularmente las escuelas de ingeniería han reconocido la necesidad que sus estudiantes tienen de adquirir las competencias requeridas para participar de forma efectiva en equipos de colaboración global. Muchas de ellas entienden el uso de proyectos colaborativos como experiencias de aprendizaje que permitirán que sus estudiantes sean más competitivos en un mundo global (Esparragoza y Devon, 2005; Esparragoza, et al. 2007; Maury-Ramírez, Pinzón, y Esparragoza, 2008). Estos tipos de proyectos se desarrollan en el contexto de una experiencia de aprendizaje basada en problemas (PBL por sus siglas en inglés) pero en un entorno internacional.

Hay varios ejemplos de universidades que se han unido para trabajar juntas en proyectos de diseño de producto colaborativo de manera que los estudiantes puedan aprender sobre los beneficios y desafíos de este tipo de configuración de trabajo (Bufardi, et al. 2005; Zavbi y Tavcar, 2004; Qamhiyah y Ramond, 2005). Siguiendo este ejemplo, la Universidad Tecnológica Centroamericana (UNITEC) se ha unido al grupo colaborativo conformado por Pennsylvania State University (EEUU), Universidad Técnica Federico Santa María (Chile), Escuela Superior Politécnica del Litoral (Ecuador), Politecnico di Milano (Italia) y la Universidad EAFIT (Colombia). Este grupo, denominado "América e Italia por el Diseño", desarrolla un proyecto de diseño colaborativo multinacional una vez al año (Octubre Diciembre) en donde estudiantes en cada universidad conforman grupos que trabajan colaborativamente para resolver un problema de diseño propuesto. 


\section{Problema de investigación}

A pesar de las muchas ventajas relacionadas a trabajar en equipos multinacionales, también existen desventajas y desafíos que deben ser solventados. Entre las dificultades asociadas con tener equipos globalmente dispersos están las complejidades típicas de tener que trabajar con otros, especialmente con gente de diferentes culturas y realidades. Scott y Einstein, (2001) indican que otro problema para preocuparse es la naturaleza transitoria de los equipos globales, lo que significa que sus miembros pueden ser parte de varios equipos simultáneamente y esto puede crear problemas debido a las diferentes formas de reconocimiento y sistemas de recompensa entre los equipos. Otra dificultad tiene que ver con la forma de medir el esfuerzo individual o rendimiento de trabajo bajo esta configuración (Harvey, et al. 2004).

Todos estos desafíos antes descritos podrían llevar a disminuir la motivación de los miembros de los equipos para colaborar e interactuar con sus socios internacionales, redundando en una baja participación en el proyecto colaborativo en cuestión. Esto es un problema grave, ya que uno de los beneficios más importantes de este tipo de configuración es precisamente la activa colaboración de miembros de equipos con puntos de vista e ideas muy diferentes productos de idiosincrasias y experiencias provenientes de diferentes partes del mundo. Toda esta interacción debería lograr productos y soluciones únicamente creativas e innovadoras que logren alcanzar las metas deseadas.

En el caso de UNITEC, las experiencias con los proyectos colaborativos multinacionales han sido muy buenas, tanto para los estudiantes como para los maestros. Sin embargo, dos de los grandes desafíos que se han encontrado son como crear una interacción más efectiva entre los estudiantes que conforman los diferentes grupos multinacionales y como mantener la motivación de los estudiantes tanto en el proyecto en sí, como en su proceso de aprendizaje.

\subsection{Objetivos de investigación}

El mejorar la interacción entre los estudiantes de forma que estos tengan un flujo de información más constante, así como el conseguir incrementar su motivación debería lograr que la participación de los estudiantes en los proyectos de diseño colaborativo sea más activa y significativa. Para esto es necesario entender cuáles son las formas de comunicación entre estudiantes más eficientes, como ésta siendo la interacción con sus compañeros de equipo (nacionales e internacionales) y que cosas pueden hacerse para mejorar la experiencia que tienen en el desarrollo de los proyectos de diseño colaborativo multinacional. Por lo tanto, los objetivos de este proyecto de investigación son:

- Evaluar las interacciones entre los estudiantes participando en el proyecto de diseño colaborativo multinacional "América e Italia por el Diseño", determinando como estas interacciones afectaron el desarrollo y finalización del proyecto. 
- Proponer acciones que ayuden a lograr una participación más activa y significativa en los proyectos.

\section{Revisión Bibliográfica}

\subsection{Uso de equipos multinacionales}

Entre los cambios que la globalización de la economía ha traído se encuentran la apertura en los mercados y la libertad que las empresas tienen de ofrecer bienes y servicios, o de adquirirlos de cualquier oferente en todo el mundo. De hecho, son muchas las empresas que buscando mejorar sus niveles de competitividad han diversificado sus operaciones fabricando sus productos en países con una mano de obra barata y vendiendo en mercados en donde puedan obtener el precio de venta más alto posible (Dornier et al. 1998; Sarache et al. 2011; Seguino y Grown, 2006). Esto, adicionalmente a la serie de alianzas estratégicas, adquisiciones y fusiones producto de esta misma globalización hace que muchas empresas multinacionales tengan a su disposición un grupo de profesionales muy capaces dispersos por todo el mundo.

Si las compañías multinacionales son capaces de organizar y comunicar a sus empleados más talentosos y experimentados sin importar su localización geográfica, podrán combinar lo mejor que tienen disponible en términos de recursos humanos con un propósito específico (Zavbi y Tavcar, 2004). La ventaja obvia de esto es que dichos equipos pueden, hipotéticamente, reducir el tiempo de desarrollo, incrementar el desempeño organizacional y mejorar la participación de los empleados debido a la sinergia que puede obtenerse (Shachaf, 2008). Para poder desarrollar esta ventaja competitiva, son muchas las empresas que buscan formar equipos colaborativos multinacionales o geográficamente dispersos.

Davidow y Malone (1992) opinan que los avances en las tecnologías de la información y la comunicación (TIC) son las que han logrado que esta colaboración puede suceder en lugares geográficamente dispersos con expertos trabajando de una forma colectiva y conjunta. Una compañía no requiere tener a todos los miembros de un equipo localizados en un solo lugar, y puede utilizar todos los recursos disponibles sin importar de su localización. De hecho, a estos equipos también se les conoce como equipos virtuales globales (GVT por sus siglas en ingles), debido a que su trabajo se hace predominantemente a través de herramienta TIC electrónicas tales como el email y la video-conferencia (Hertel, et al. 2005; Lee-Kelley y Sankey, 2008; Wilczynski y Jennings, 2003).

De acuerdo a Gibson y Cohen, (2003) y Hinds, (2002) la mayoría de las grandes corporaciones usan equipos virtuales. De hecho, de acuerdo a AFW, (2002) en Hertel, et al. (2005) una encuesta entre 376 gerentes de empresas de diferentes compañías en Alemania mostro que $20 \%$ de ellos trabajan principalmente como parte de equipos virtuales y alrededor de $40 \%$ de ellos había trabajado al menos temporalmente en ellos. La misma tendencia es 
verdadera en la industria de fabricación de automóviles (May y Carter, 2001), banca (LeeKelley y Sankey, 2008) y otros rubros ya que el uso de equipos virtuales globales (multinacionales o geográficamente dispersos) no es exclusivo a la ingeniera y los negocios, sino también a las ciencias en general (Franzoni y Sauermann, 2014).

\subsection{Evaluando interacciones en equipos geográficamente dispersos}

Uno de los requisitos para que un equipo virtual global (geográficamente disperso) sea exitoso es que los miembros del equipo sean capaces y estén comprometidos (Ebrahim, Ahmed, y Taha, 2009; Goodbody, 2005). Las competencias requeridas de cada miembro de los equipos dependerán en las metas específicas del equipo. La confianza entre los compañeros de equipo requerida para fomentar el espíritu de cooperación necesario para cumplir con los objetivos del equipo puede ser construido sobre el constante buen comportamiento de los individuos (Robert, Jr., Dennis, y Hung, 2009). Por el contrario, el mal comportamiento tal como la falta de comunicación, el bajo nivel de responsabilidad individual, la falta de compromiso hacia el trabajo de calidad, etc. genera poca confianza entre los miembros del equipo y pone en peligro la eficacia del trabajo en equipo (Tseng y Yeh, 2013). Por lo tanto, el evaluar la conducta de los miembros del equipo en sus interacciones y el cumplimiento de sus tareas asignadas es muy importante.

Para todo equipo de trabajo, la buena comunicación es crítica para tener el éxito esperado. Por lo tanto, otro requisito para que los equipos geográficamente dispersos sean exitosos es tener herramientas correctas para que sus miembros puedan trabajar colaborativamente (LeeKelley y Sankey, 2008). De acuerdo a Gibson y Cohen (2003), esta comunicación se realiza más a menudo a través email o llamadas por conferencia (tecnología de primera generación). Sin embargo, como Lee-Kelley, et al. (2004) y Maznevski y Chudoba, (2000) explican, cierto nivel de interacción cara-a-cara es también necesaria para mejorar la comunicación y confianza. Este tipo de comunicación puede hacerse de forma física o usando herramientas TIC (tecnologías de segunda y tercera generación) tales como video conferencias y espacios web de trabajo compartidos habilitados a través del internet.

A pesar que ciertos investigadores sugieren que las interacciones cara-a-cara pueden ser contraproducentes al enfatizar las diferencias entre los miembros y producir sesgo afectando futuras comunicaciones entre compañeros de equipo (Bjørn y Ngwenyama, 2009), la mayoría de la investigación parece apoyar el efecto positivo que este tipo de comunicación tiene en el desempeño de los equipos virtuales globales (Hosseini y Chileshe, 2013). La calidad de esta comunicación se ve afectada por el tipo de TIC que se usa para compartir conocimiento (ideas, conceptos, modelos) y por la infraestructura disponible para apoyarla (Karoui, Gürkan, y Dudezert, 2010).

Finalmente, otro factor que debe tomarse en consideración es la diferencia de tiempo entre los miembros del equipo, lo que requiere de capacidades de comunicación asíncrona, 
diferencias en idioma y cultura, y la necesidad de medir la contribución y participación de los miembros del equipo (Dekker, Rutte, y Van den Berg, 2008; Zimmermann, 2011). Debido a la importancia de esta interacción, universidades tales como Syracuse University, Hong Kong University y Lancaster University han estado trabajando en proyectos de investigación con el propósito de desarrollar sistemas de diseño colaborativo, habilitados por la web y herramientas bajo una arquitectura cliente-servidor. De igual forma, Carnegie Mellon University, University of California Berkeley, University of Tennessee en Chattanooga y Stanford University han estado trabajando en herramientas semejantes usando un enfoque de diseño colaborativo basado en agentes (Wang, et al. 2002).

\subsection{Proyectos colaborativos universitarios}

Un proyecto colaborativo académico es una estrategia de enseñanza diseñada para animar a los estudiantes a trabajar en grupos geográficamente dispersos hacia el logro de una meta. Esta estrategia crea un ambiente de aprendizaje en donde el conocimiento es compartido o transmitido entre compañeros de equipo (Brindley y Walti, 2009). De acuerdo a Palloff y Pratt, (2005), el aprendizaje colaborativo tiene beneficios pedagógicos tales como el desarrollo de habilidades de pensamiento crítico, la co-creación de conocimiento y significado, reflexión y aprendizaje transformativo. Siemens, (2002) sugiere que estos beneficios se logran a través de un continuo de cuatro etapas compuesto de comunicación (gente hablando y discutiendo), colaboración (personas que comparten ideas y recursos, así como trabajando juntos), cooperación (gente haciendo cosas juntos) y la comunidad (personas con un objetivo común).

Como se explicó previamente, las universidades han reconocido la necesidad que sus estudiantes tienen de adquirir los tipos de competencias que son necesarias para participar de forma efectiva en equipos de colaboración global. Al desarrollar estas competencias los estudiantes se preparan para poder trabajar y ser competitivos en un mundo global (MauryRamírez, Pinzón, y Esparragoza, 2008). Son varias las universidades que se han unido para trabajar juntas en proyectos de diseño de producto colaborativo, mientras otras se han enfocado en el desarrollo de herramientas que faciliten la interacción y colaboración entre equipos.

Un ejemplo de colaboraciones entre universidades es el Proyecto Global Europeo de Realización de Productos (E-GPR por sus siglas en ingles) que juntó al Instituto Federal Suizo de Tecnología de Lausanne, la Universidad Técnica de Delft (Holanda) y la Universidad de Ljubljana (Eslovenia). Los objetivos del E-GPR eran exponer a los estudiantes a la experiencia de resolver un proyecto global de desarrollo de producto usando el conocimiento adquirido durante la clase, así como el conocimiento aprendido en clases previas y la información y datos adquiridos de socios industriales (Bufardi, et al. 2005; Zavbi y Tavcar, 2004). Otros ejemplos incluyen el CADAU (Computer Aided Design Across Universities), que es una colaboración internacional entre cursos de diseño asistido por 
computadora de la Universidad del Estado de Iowa (ISU) en los Estados Unidos y la Universidad de Tecnología de Compiegne (UTC) en Francia (Qamhiyah y Ramond, 2005) y el ICON (Institutional Collaboration Over Networks) entre estudiantes de clases de Diseño de Producto en la Universidad de Stratchclyde y la Universidad de Glasgow, ambas en Escocia (Sclater, Grierson, Ion, y MacGregor, 2001) entre otros.

Debido a la importancia que tiene la interacción entre miembros de equipo, la Universidad de Syracuse, Universidad de Hong Kong y la Universidad de Lancaster han estado trabajando en proyectos de investigación con el objetivo de desarrollar sistemas y herramientas de diseño de colaboración basadas en la web en una arquitectura cliente-servidor. Del mismo modo, la Universidad Carnegie Mellon, la Universidad de California en Berkeley, la Universidad de Tennessee en Chattanooga y la Universidad de Stanford han estado trabajando en herramientas similares usando un enfoque de diseño colaborativo basado en agentes. (Wang, Weiming, Xie, Neelamkavil, y Pardasani, 2002).

\subsection{Proyecto de diseño colaborativo: "América e Italia por el diseño"}

Uno de los proyectos de diseño colaborativo que se ha desarrollado entre instituciones del Consorcio Latinoamericano y del Caribe de Instituciones de Ingeniería (LACCEI por sus siglas en inglés) es el denominado “América e Italia por el Diseño" (Esparragoza y Devon, 2005; Esparragoza, et al. 2007; Maury-Ramírez, Pinzón, y Esparragoza, 2008).

El proceso de trabajo y colaboración utilizado durante el trimestre se muestra en la Figura 1. Este proyecto se realiza durante el último semestre del año (iniciando en octubre) con grupos de estudiantes que trabajan independientemente en desarrollar una propuesta de diseño para un problema común, pero se les requiere compartir información e ideas con sus socios internacionales para mejorar su solución y propuesta final (Esparragoza, et al. 2014). Por ejemplo, el desafío durante el proyecto del 2013 fue diseñar un triciclo portátil y plegable para niños en edades entre 3 y 5 años con una pierna amputada. Los requerimientos iniciales del triciclo eran: económicamente accesible, fácil de transportar, plegable, fácil de usar aun para niños con fortaleza limitada en las piernas y brazos.

Las redes de colaboración se forman creando clústeres de dos a cinco equipos de diferentes universidades que se agrupan para trabajar colaborativamente en la resolución de un problema de diseño. La Figura 2 muestra la red colaborativa que se conformó durante el año 2013, compuesta de seis clústeres con 28 equipos de seis países diferentes. Las universidades participantes fueron mencionadas en la introducción de este documento, entre las cuales está Unitec.

Entre las herramientas disponibles para la comunicación esta ANGEL (sistema de administración de clases de Penn State), Adobe Connect Meeting (dirección especifica provista por Penn State para cada clúster) y otras herramientas de uso común. 


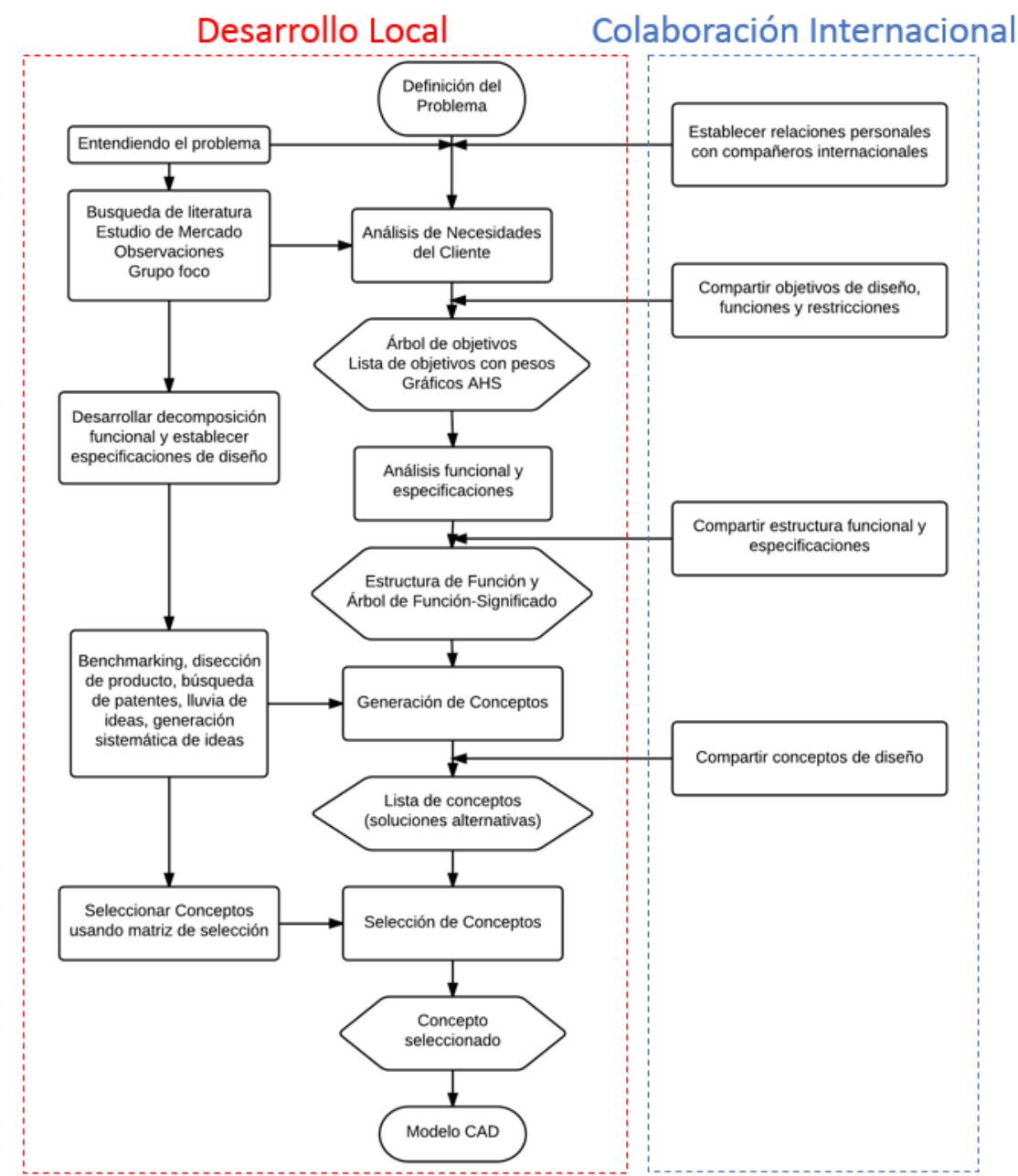

Figura1. Proceso de diseño para el proyecto colaborativo
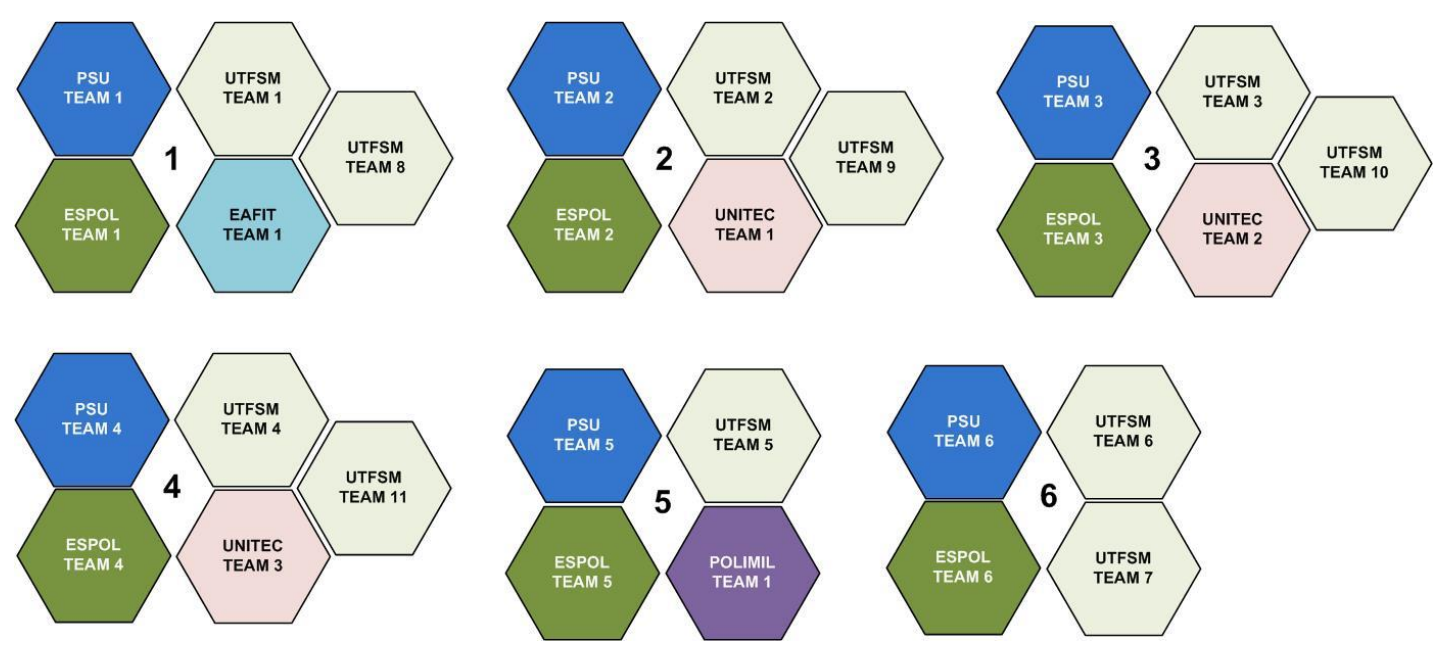

Figura 2. Red de colaboración para proyecto de diseño colaborativo del 2013 


\section{Metodología}

En vista que el proyecto de diseño de productos colaborativo multinacional es usado como herramienta educativa, este requiere de una evaluación para reunir información sobre el proceso de enseñanza-aprendizaje de los estudiantes y usar dicha información para realizar mejoras (Steward, Mickelson, y Brumm, 2005). En este proyecto de investigación se evaluó el valor de la interacción social en el flujo de información durante el proyecto colaborativo realizado durante el año 2013 y el impacto que esta tuvo en completar exitosamente el proyecto. Usando esta información se buscó encontrar áreas de mejora para futuras ediciones del proyecto colaborativo.

Para poder evaluar la importancia que tiene la interacción social entre los grupos conformados en la conclusión exitosa del proyecto asignado, se realizó una encuesta entre los alumnos una vez que hubieron terminado la clase. La encuesta fue diseñada para ser llenada en línea usando un portal ubicado en www.qualtrics.com. La participación fue voluntaria, aunque se buscó animar a todos a participar. Las respuestas fueron anónimas para fomentar respuestas verdaderas entro los estudiantes.

\subsection{Recolección de datos}

Los datos usados fueron recolectados de encuestas realizadas a los alumnos participando en el proyecto de diseño colaborativo global. De todos los estudiantes inscritos, 103 contestaron la encuesta en línea. Las preguntas que se hicieron pueden dividirse en cinco grandes categorías: Demografía (género y localización), Intensidad de la interacción (cuan a menudo se interactuó con los socios internacionales usando TICs, cuan a menudo se participó en las reuniones programadas y cuantas horas se trabajó con ellos), Tipo de interacción usada (cuales fueron los medios de comunicación más usados y el tipo de información de contacto compartida), Utilidad de la interacción (preguntas que se enfocaron en la cantidad de la interacción realizada e información recibida, la calidad de la interacción y de la información entregada y recibida, así como la utilidad de la información y de la experiencia para la conclusión del proyecto) y Preguntas abiertas (que hicieron bien como equipo, que problemas encontraron y acciones de mejora propuestas).

\subsection{Confiablidad de la encuesta}

Las preguntas fueron validadas entre todos los maestros participando en el proyecto para asegurar que todos los elementos que se querían estudiar fueran tomados en cuenta. El instrumento fue diseñado con varias preguntas de selección múltiple de tipo nominal y ordinal (demografía, intensidad de interacción y tipo de interacción usada) y preguntas abiertas que no se prestan para revisar fiabilidad. Sin embargo, las preguntas sobre la utilidad de la interacción si fueron diseñadas con preguntas en una escala Likert que si permiten medir fiabilidad y hacer análisis de factores. 
Tabla 1. Matriz de componentes rotados

\begin{tabular}{|c|c|c|c|}
\hline & Factor 1 & Factor 2 & Factor 3 \\
\hline $\begin{array}{c}\text { Resultados de las interacciones con socios } \\
\text { internacionales }\end{array}$ & $\begin{array}{l}\text { Utilidad } \\
\text { académica de } \\
\text { la experiencia }\end{array}$ & $\begin{array}{l}\text { Calidad de la } \\
\text { interacción e } \\
\text { información }\end{array}$ & $\begin{array}{l}\text { Utilidad de la } \\
\text { interacción para } \\
\text { finalizar el } \\
\text { proyecto }\end{array}$ \\
\hline $\begin{array}{l}\text { Mi experiencia académica (know-how) era adecuada para enfrentar esta } \\
\text { experiencia colaborativa }\end{array}$ & 0.807 & & \\
\hline Esta experiencia colaborativa me motivó a trabajar en el proyecto & 0.795 & & \\
\hline $\begin{array}{l}\text { La cantidad de tiempo provista antes de las reuniones fue adecuada para } \\
\text { desarrollar cada asignación del proyecto colaborativo }\end{array}$ & 0.721 & & \\
\hline $\begin{array}{l}\text { Mis compañeros internacionales estaban siempre preparados para las } \\
\text { reuniones, participando activamente y compartiendo información a tiempo }\end{array}$ & 0.695 & & \\
\hline $\begin{array}{l}\text { Esta experiencia colaborativa me ayudó a mejorar mis habilidades } \\
\text { profesionales }\end{array}$ & 0.658 & & \\
\hline Esta experiencia colaborativa le ayudó a mi equipo a terminar el proyecto & 0.646 & & \\
\hline $\begin{array}{l}\text { La actividad colaborativa me ha permitido desarrollar el diseño por medio } \\
\text { de un método sistemático para resolver problemas }\end{array}$ & 0.554 & & \\
\hline $\begin{array}{l}\text { La cantidad de información recibida de nuestros compañeros internacionales } \\
\text { fue adecuada para el proyecto colaborativo }\end{array}$ & & 0.788 & \\
\hline $\begin{array}{l}\text { Mi equipo estaba siempre preparado para las reuniones, participó } \\
\text { activamente y compartió la información a tiempo }\end{array}$ & & 0.747 & \\
\hline $\begin{array}{l}\text { Todos los equipos en nuestro clúster contribuyeron equitativamente al } \\
\text { proyecto colaborativo }\end{array}$ & & 0.640 & \\
\hline $\begin{array}{l}\text { La calidad de información recibida de nuestros compañeros internacionales } \\
\text { fue adecuada para el proyecto colaborativo }\end{array}$ & & 0.635 & \\
\hline $\begin{array}{l}\text { La interacción personal fue importante para construir confianza y facilitar } \\
\text { comunicación en el proyecto colaborativo multinacional }\end{array}$ & & 0.629 & \\
\hline $\begin{array}{l}\text { La cantidad de tiempo provista para la reunión (video-conferencia) fue } \\
\text { adecuada para el proyecto colaborativo }\end{array}$ & & 0.475 & \\
\hline $\begin{array}{l}\text { La interacción personal no fue adecuada para mantener un flujo de } \\
\text { información para el proyecto colaborativo multinacional }\end{array}$ & & & 0.788 \\
\hline $\begin{array}{l}\text { La interacción personal contribuyó al flujo de información para el proyecto } \\
\text { colaborativo multinacional }\end{array}$ & & & 0.735 \\
\hline $\begin{array}{l}\text { La cantidad de información entregada a los compañeros internacionales fue } \\
\text { adecuada para el proyecto colaborativo }\end{array}$ & & & 0.732 \\
\hline $\begin{array}{l}\text { El método que utilice para enfrentar el diseño de producto fue mejorado por } \\
\text { esta experiencia colaborativa }\end{array}$ & & & 0.571 \\
\hline $\begin{array}{l}\text { La información recibida de nuestros compañeros internacionales fue valiosa } \\
\text { para completar el proyecto }\end{array}$ & & & 0.563 \\
\hline
\end{tabular}

Se realizó una prueba de fiabilidad usando Alfa de Cronbach, que mide la consistencia interna de un constructo. Inicialmente se revisó la fiabilidad de la sección del instrumento que buscaba medir la utilidad de la interacción (18 preguntas) obteniendo un valor de 0.933 (Tabla 2) lo que excedió el nivel de corte de 0.60. Luego las variables (preguntas) fueron 
analizadas mediante un análisis exploratorio de factores (con rotación Varimax) para examinar si los ítems compartían un único factor subyacente. El resultado mostró que existen 3 factores principales alrededor de los cuales se pueden agrupar las variables (Tabla 1). Finalmente se revisó la fiabilidad de cada uno de estos factores utilizando el análisis de Alfa de Cronbach (Tabla 2).

Tabla 2. Resumen del análisis de fiabilidad de los constructos

\begin{tabular}{|l|c|}
\hline \multicolumn{1}{|c|}{ Constructo } & Alfa de Cronbach \\
\hline Utilidad de la interacción & 0,933 \\
\hline Aprovechamiento académico & 0,909 \\
\hline Calidad de información & 0,836 \\
\hline Utilidad para el proyecto & 0,838 \\
\hline
\end{tabular}

\section{Resultados y Análisis}

\subsection{Demografía}

Como se explicó inicialmente, los participantes en el proyecto son estudiantes de ingeniería de clases de diseño de productos de 6 universidades localizadas en países diferentes. Como se observa en la Figura 3, la mayoría de los estudiantes que contestaron la encuesta estaban en Chile, seguidos por Ecuador, Estados Unidos y Honduras. De estos estudiantes el 83\% eran hombres y el $17 \%$ restante mujeres.

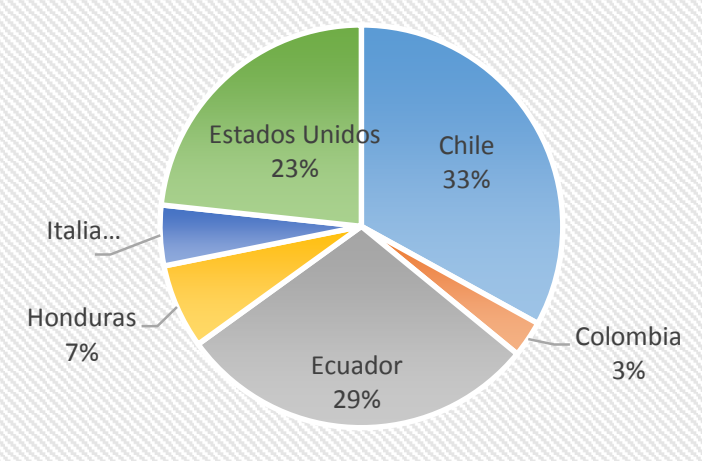

Figura 3. Localización de estudiantes encuestados por país

\subsection{Intensidad de la interacción}

En vista que el objetivo principal de este estudio es revisar el efecto que tiene la interacción social de los alumnos en la conclusión exitosa del proyecto, se revisó la frecuencia de las 
interacciones con los socios internacionales mediante el uso de tecnologías de la información y comunicación (TICs) tales como el email, la video-conferencia, etc. (Figura 4 y 5) así como la cantidad de horas dedicadas a este tipo de interacción (Figura 6).

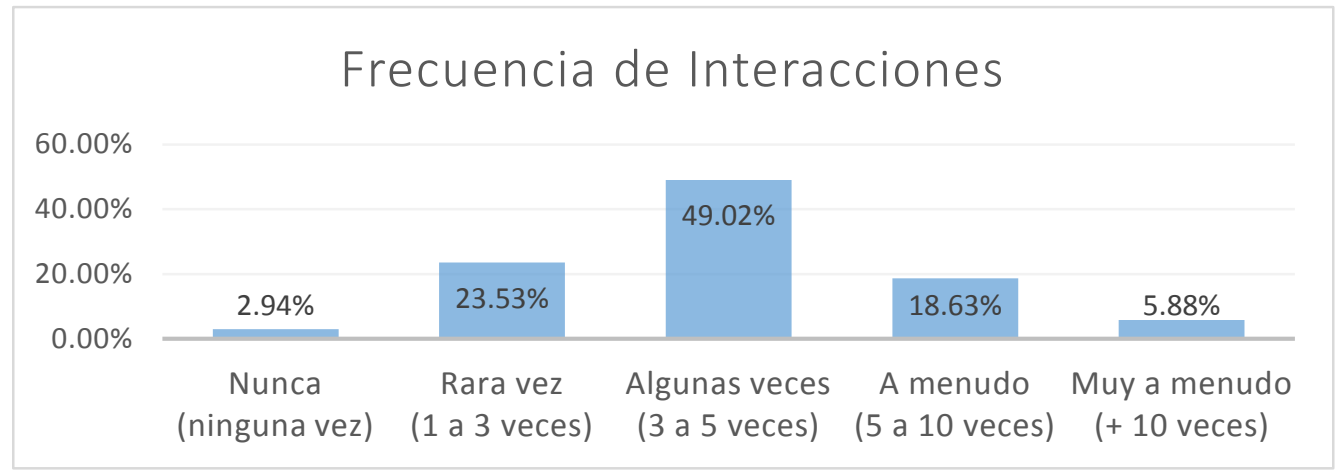

Figura 4. Cuan a menudo interactuó con sus socios internacionales usando TICs

Como se puede observar en la Figura 4, las interacciones no fueron muy frecuentes (aproximadamente un 75\% de los estudiantes reportaron menos de 5 interacciones en todo el trimestre). Si se toma en cuenta que todos los equipos tenían 4 reuniones programadas por video-conferencia, y que la mayoría participo en ellas (Figura 5), se puede asumir que la mayoría no tuvieron más interacciones que las programadas y obligatorias.

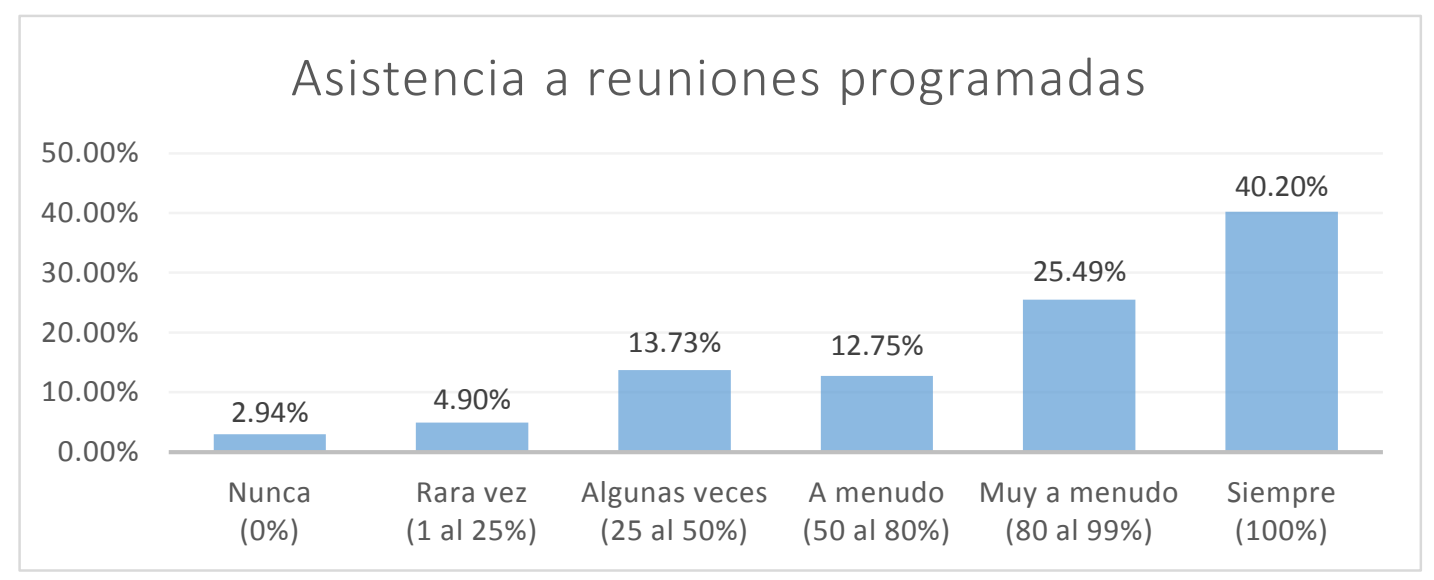

Figura 5. Cuan a menudo asistió a sus reuniones programadas por video-conferencia

En cuanto a la duración de estas interacciones, Como se puede observar en la Figura 6 la mayoría de estas fue de entre 1 y 3 horas, con un 10\% reportando no haberse reunido para nada y un $5 \%$ expresando haberse reunido más de 3 horas a la semana. 


\section{Horas de trabajo semanal con socios internacionales}

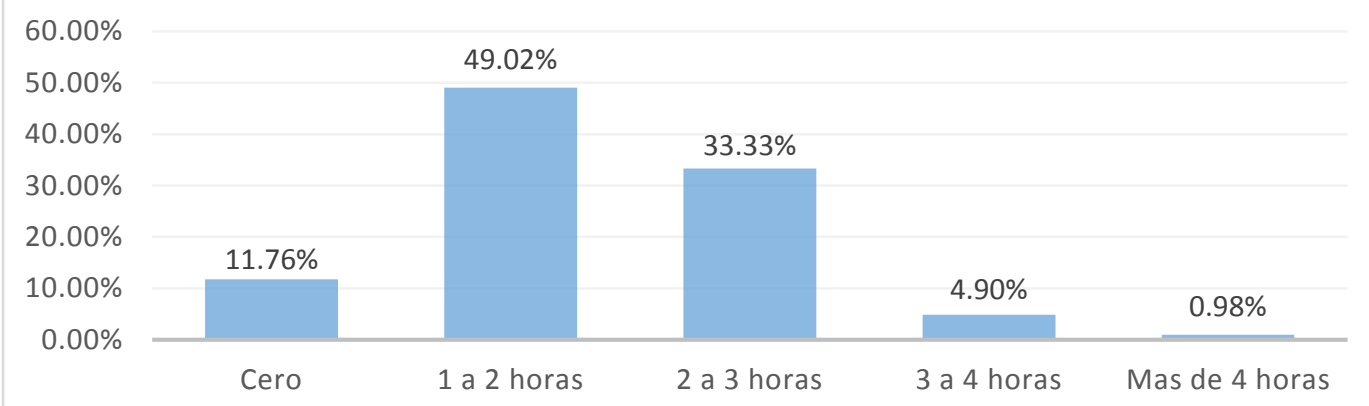

Figura 6. Horas a la semana que paso trabajando con sus socios internacionales

\subsection{Tipo de interacción usada}

Un equipo aumentará sus interacciones si el medio de comunicación es efectivo (Lee-Kelley y Sankey, 2008). Por esta causa se buscó investigar cual es el medio de interacción y comunicación preferido por los estudiantes.

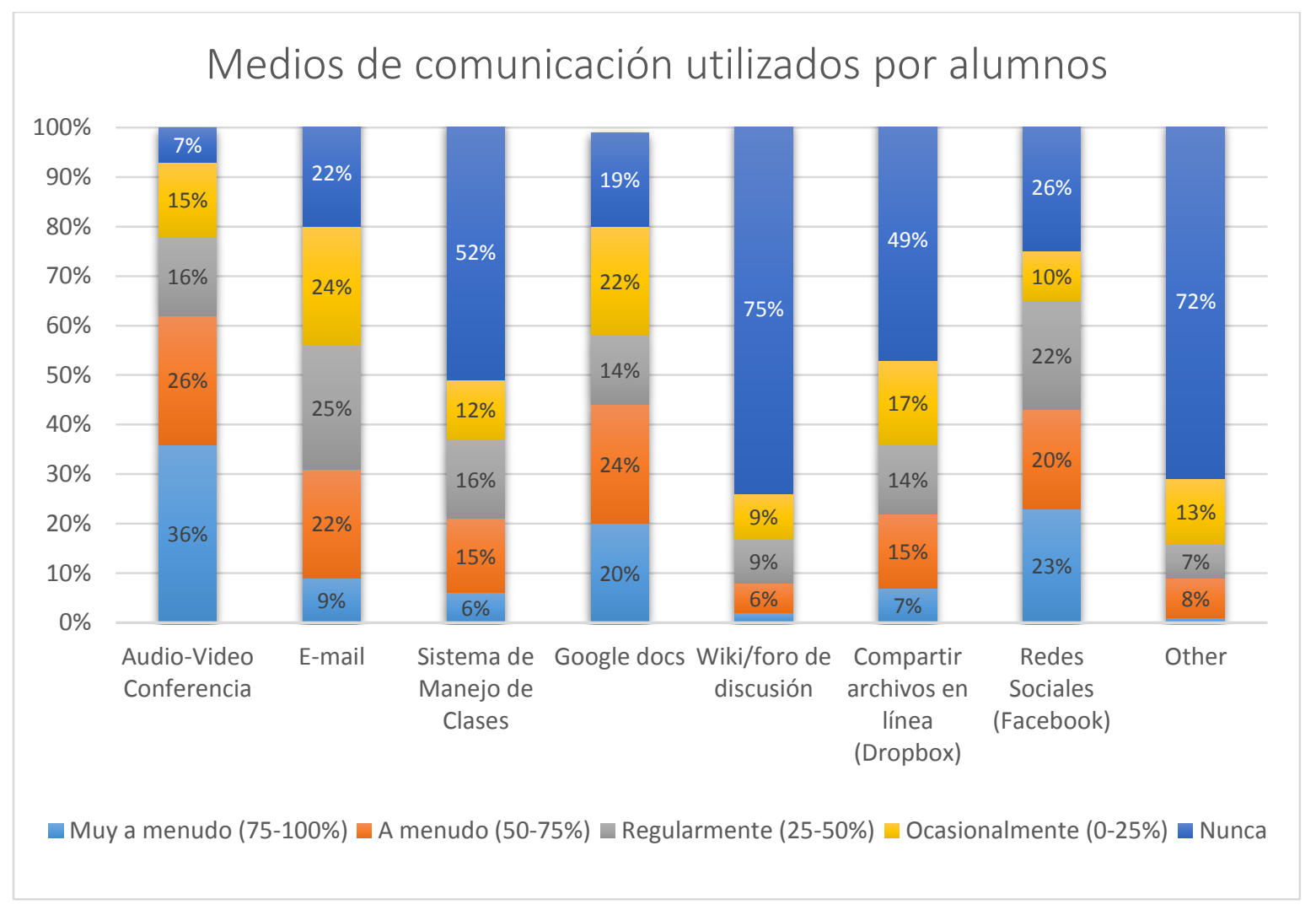

Figura 7. Medios de comunicación más usados por alumnos 
La Figura 7 muestra las respuestas de los alumnos a la pregunta ¿Cuál fue el medio que utilizó para su interacción con los socios internacionales y en qué porcentaje? En ella se puede observar que la video-audio conferencia fue el medio de comunicación más usado para las interacciones con los socios internacionales. Luego se destaca el uso de las redes sociales tales como Facebook y en menor escala el uso del correo electrónico. También es interesante el bajo uso de herramientas para compartir documentos tales como Dropbox y su sustitución por Google Docs y probablemente los adjuntos en correos electrónicos. Otros medios de comunicación usados fueron Twitter, WhatsApp, Skype y mensajes de texto.

En la Figura 8 se nota la importancia que tiene el uso de las redes sociales en la comunicación durante situaciones de trabajo en equipo colaborativo multinacional junto con el correo electrónico, especialmente entre jóvenes ingenieros.

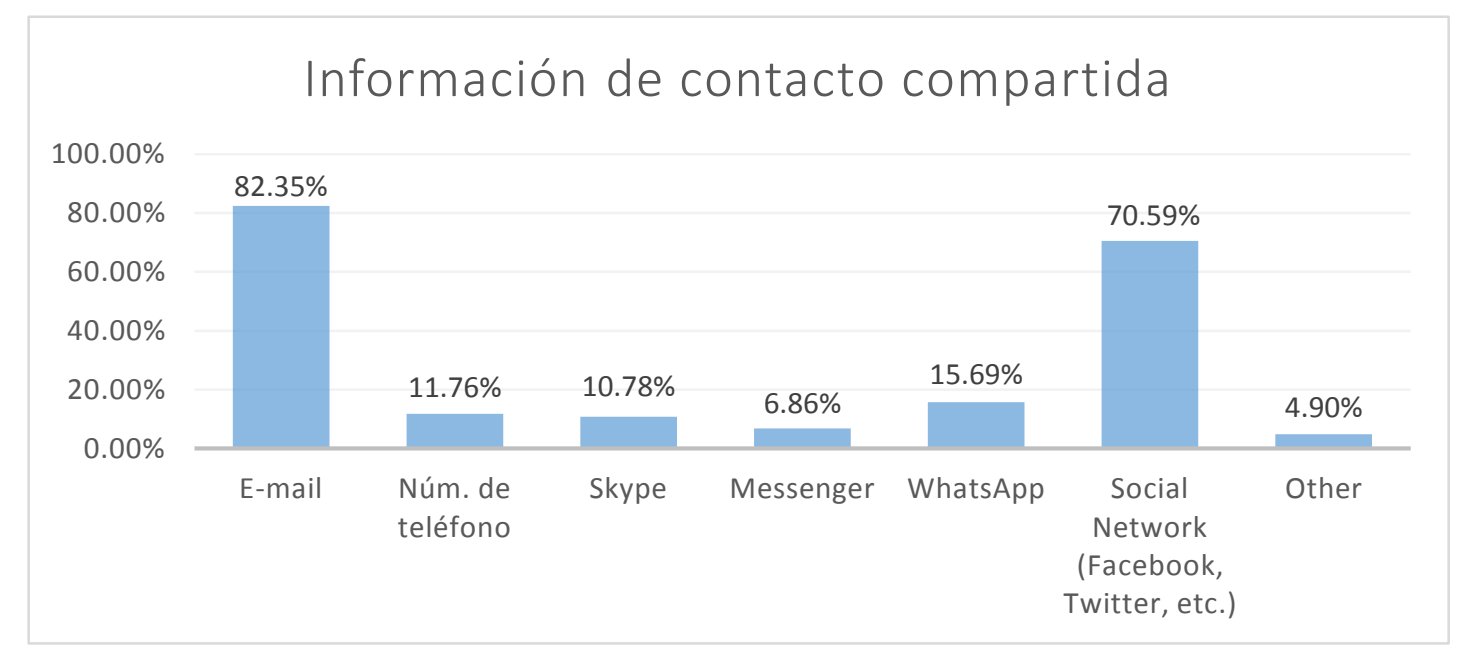

Figura 8. Información de contacto compartida con socios internacionales.

\subsection{Utilidad de la interacción}

La comunicación e interacción entre equipos que conforman un clúster debería de mejorar la calidad final del proyecto a desarrollar al tener acceso a más ideas y diferentes perspectivas para resolver el problema en cuestión. De las encuestas se encontró que a pesar que las interacciones no fueron muy frecuentes, si fueron lo suficientemente eficientes como para compartir información que era muy importante para completar el proyecto de diseño colaborativo. En la Figura 9 se observa que aproximadamente un $80 \%$ de los estudiantes compartió las necesidades del cliente y los objetivos de diseño, alrededor de un $70 \%$ las funciones del producto e ideas de concepto, y cerca de un $60 \%$ las restricciones de diseño y las especificaciones del producto. 


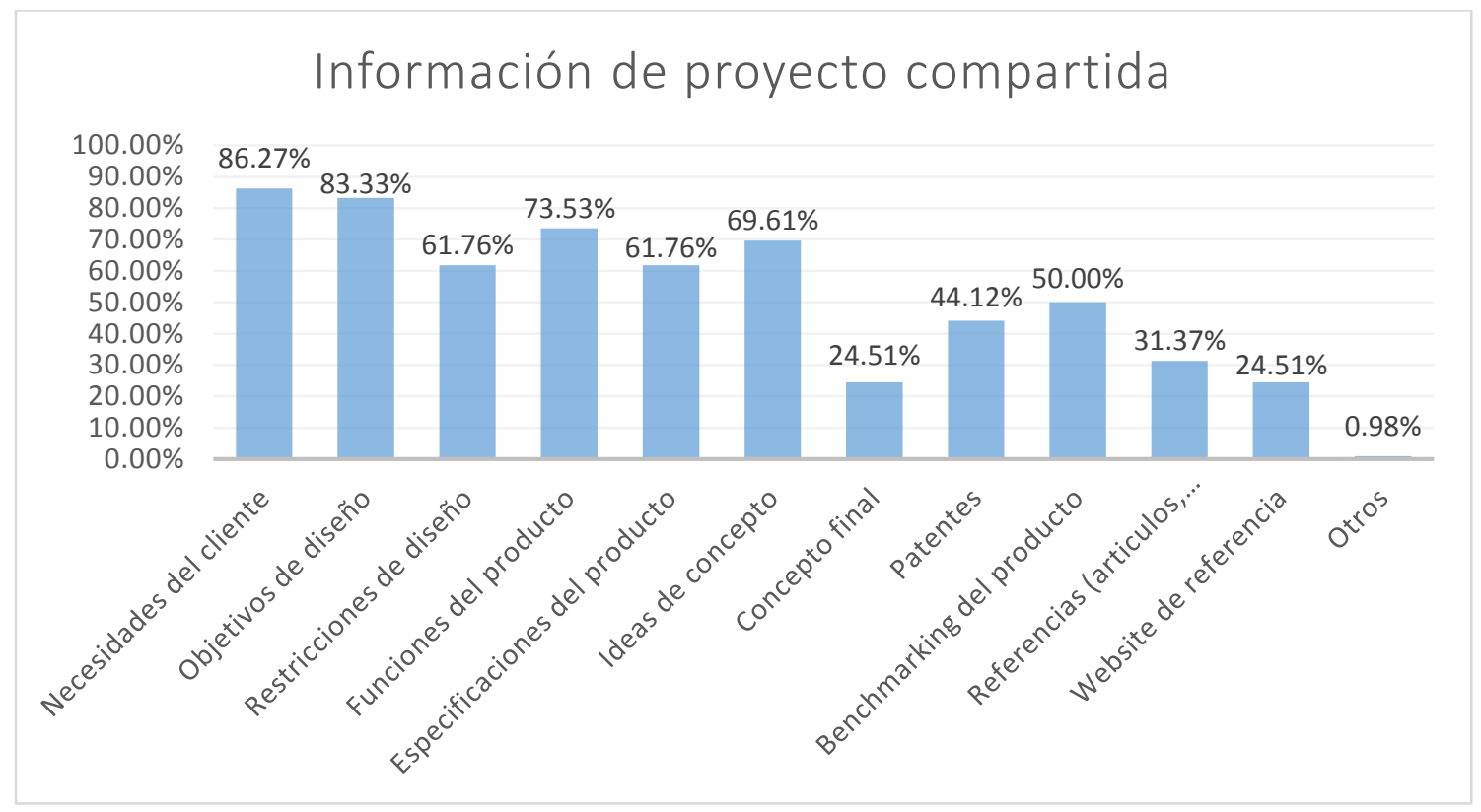

Figura 9. Información del proyecto compartida con socios internacionales

La información que se compartió en menor escala fueron referencias de ayuda y el concepto final. Sin embargo, a pesar que se compartió bastante información que era necesaria para terminar el proyecto, de acuerdo a los encuestados, la información recibida de sus socios internacionales no contribuyo significativamente a completar el proyecto (Figura 10). Pareciera que la información compartida no fue tan relevante, probablemente por problemas con la calidad de la información enviada o por haberse entregado fuera del tiempo requerido.

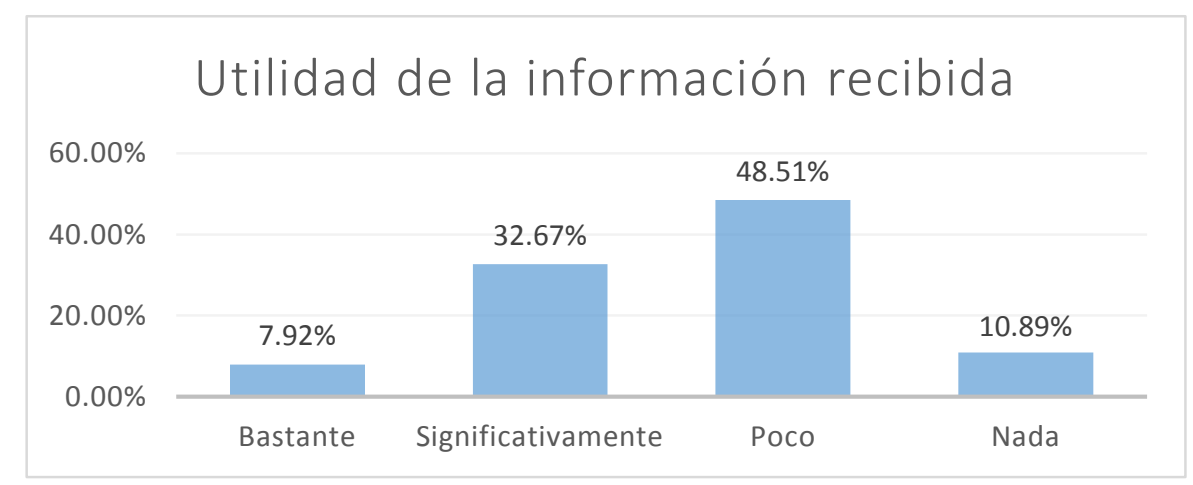

Figura 10. Utilidad de la información recibida para terminar el proyecto 
En la Figura 11 se presentan los resultados de 18 preguntas relacionadas con la cantidad, calidad y utilidad de la información compartida entre socios internacionales y su interacción. De estas respuestas se recoge que el $58 \%$ están de acuerdo que la cantidad de información compartida y recibida fue la adecuada. Igualmente, casi la mitad de los participantes (47\%) opinan que la cantidad de tiempo provista para las reuniones y entre reuniones (tiempo de preparación) era el apropiado. Sin embargo, es interesante que menos de la mitad (48\%) opinan que la calidad de la información recibida fue la apropiada.

Como preguntas adicionales para revisar la calidad, se indagó sobre la interacción personal y su rol en construir confianza, mejorar el flujo de información y facilitar la comunicación, ya que esto agrega a la calidad de la información compartida. Como se observa en la Figura 11, en el caso de la importancia de la interacción personal con los socios internacionales, más del $60 \%$ están de acuerdo o totalmente de acuerdo que esta fue importante para construir confianza y para mejorar el flujo de información en el proyecto. Sobre la preparación de los equipos para sus reuniones y su interacción con sus socios internacionales, el $79 \%$ están de acuerdo o totalmente de acuerdo que su equipo siempre estaba preparado y participó activamente en las reuniones programadas, compartiendo información a tiempo.

Referente a la utilidad, en general los estudiantes consideraron que el proyecto fue una experiencia valiosa tanto para completar su proyecto de diseño como para aprender nuevas habilidades profesionales. El 53\% están de acuerdo que el método para diseñar su producto fue mejorado por causa de los insumos recibidos en la actividad colaborativa. Un $48 \%$ de los estudiantes esta también de acuerdo que la actividad colaborativa permitió tener una metodología estructurada para resolver los problemas establecidos en la clase. Valores más bajos se obtuvieron sobre lo valioso de la información recibida de los socios internacionales, lo cual confirma la opinión vertida previamente sobre la baja calidad de los insumos obtenidos y de la intrascendente de la información compartida (Figura 11). Sin embargo, a pesar que los aportes parece que no fueron tan útiles, la experiencia si lo fue ya que un $66 \%$ afirman estar de acuerdo o totalmente de acuerdo que sus habilidades profesionales se incrementaron y un $61 \%$ dicen igualmente estar de acuerdo o totalmente de acuerdo que la experiencia colaborativa les motivo a trabajar en el proyecto y culminarlo.

La última pregunta de este grupo de preguntas se enfoca en averiguar si los alumnos consideraban tener los pre-requisitos necesarios para enfrentar el proyecto de diseño colaborativo con éxito. Un 70\% dijo tener los conocimientos académicos necesarios (knowhow) al afrontar la clase. 


\section{Evaluación de la interacción entre estudiantes}

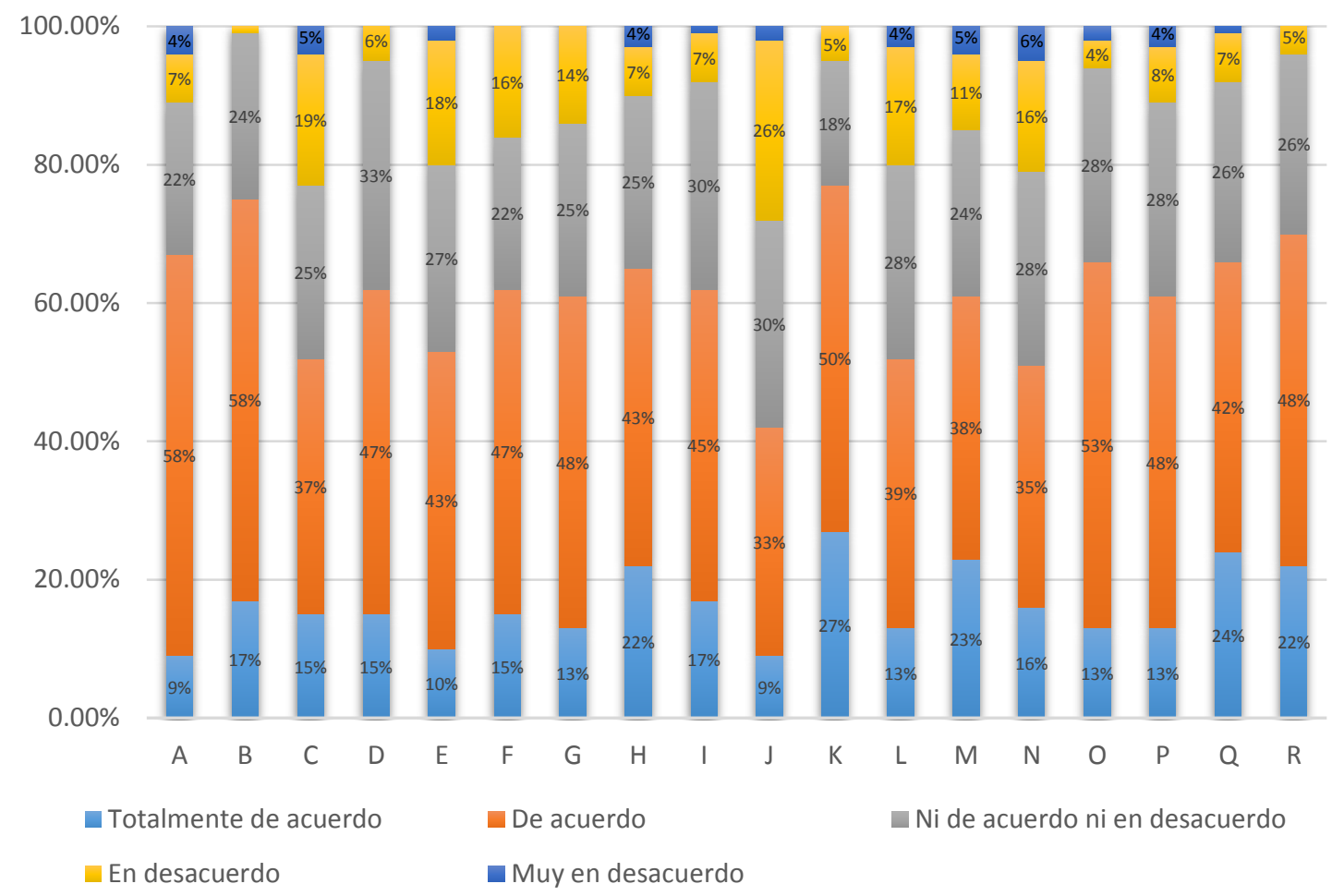

\section{Leyenda: $\quad$ Figura 11. Evaluación de la interacción entre estudiante}

A: La cantidad de información recibida de nuestros socios internacionales fue adecuada para el proyecto colaborativo.

B: La cantidad de información que proveímos a nuestros socios internacionales fue adecuada para el proyecto colaborativo

C: Todos los equipos en nuestro clúster contribuyeron equitativamente al proyecto colaborativo

D: La cantidad de tiempo provista antes de las reuniones fue adecuada para desarrollar cada asignación del proyecto colaborativo

E: Mis socios internacionales estaban siempre preparados para las reuniones, participando activamente y compartiendo información a tiempo

F: La cantidad de tiempo provista para la reunión (video-conferencia) fue adecuada para el proyecto colaborativo

G: La calidad de información recibida de nuestros socios internacionales fue adecuada para el proyecto colaborativo

H: La interacción personal fue importante para construir confianza y facilitar comunicación en el proyecto colaborativo multinacional

I: La interacción personal contribuyó al flujo de información para el proyecto colaborativo multinacional

J: La interacción personal no fue adecuada para mantener un flujo de información para el proyecto colaborativo multinacional

K: Mi equipo estaba siempre preparado para las reuniones, participó activamente y compartió la información a tiempo

L: La información recibida de nuestros socios internacionales fue valiosa para completar el Proyecto

M: Esta experiencia colaborativa me motivó a trabajar en el Proyecto

$\mathrm{N}$ : Esta experiencia colaborativa le ayudó a mi equipo a terminar el Proyecto

O: El método que utilice para enfrentar el diseño de producto fue mejorado por esta experiencia colaborativa

P: La actividad colaborativa me ha permitido desarrollar el diseño por medio de un método 


\subsection{Preguntas abiertas}

Tres preguntas abiertas fueron hechas a los estudiantes para tratar de capturar áreas de mejora para futuros proyectos colaborativos multinacionales. Algunos ejemplos de las respuestas recibidas son las siguientes:

¿Cosas que usted y/o su equipo hicieron bien durante esta experiencia colaborativa?

Puntualidad, compartir herramientas, honestidad, responsabilidad, calidad de trabajo, transferir ideas, compartir investigación hecha, trabajo en equipo, organización, presentar todos los avances, asistir a todas las reuniones (aun si era en un día festivo), usar el inglés para mejorar la comunicación, interactuar con otros equipos.

¿Problemas que usted y/o su equipo tuvieron interactuando con socios internacionales?

Barrera del idioma, equipos no compartían diseños, limitaciones de tiempo, mediocridad, irresponsabilidad, falta de motivación, pobre conexión de internet, falta de cámara, falta de puntualidad a la hora de las conferencias, falta de confianza, pobre comunicación.

¿Acciones específicas que ayudarían la interacción con sus socios internacionales?

Mejor comunicación, más tiempo entre entregas, horarios de video-conferencia más flexibles, grupos de un mismo país, más reuniones programadas, usar redes sociales, evitar video-conferencias y hacer contacto no-formal mediante e-mail o Dropbox, poder hablar con los socios internacionales, buena conexión de internet, equipos de trabajo con las mismas instrucciones de trabajo, noción básica del idioma de los otros socios, más tiempo para la video-conferencia, más formas de comunicación sincrónica.

\section{Conclusiones}

Este trabajo ha revisado el nivel de interacción que han tenido los alumnos participando en el proyecto de diseño colaborativo multinacional denominado "América e Italia por el Diseño". De las respuestas recibidas se puede concluir que el nivel de interacción entre los estudiantes y sus socios internacionales es bajo, ya que a pesar que la mitad de los estudiantes interactuó entre 3 y 5 veces durante el curso (con el resto de interacciones distribuidas de forma casi normal), 4 de estas eran las reuniones obligatorias de clúster. Adicionalmente estas interacciones eran cortas ya que el trabajo colaborativo semanal tiene una media de 2 horas, sesgado positivamente (hacia cero).

Observando las respuestas a otras preguntas dentro de la encuesta se puede deducir que posibles causas de esta baja participación son el desánimo entre los alumnos producto de problemas culturales (falta de seriedad, impuntualidad, falta de confianza), tecnológicos (falta de equipo adecuado, fallas de internet), de comunicación (desafíos con el idioma, horarios incompatibles, falta de habilidad de expresión y de comunicación asincrónica), así como malas experiencias con la calidad de los insumos obtenidos en dichas reuniones. De hecho, una de las revelaciones más interesantes de este estudio es la percepción de los 
participantes que las aportaciones de los socios internacionales son de baja calidad, lo que hizo que consideraran que la utilidad de estos insumos es muy baja.

A pesar de la baja interacción entre socios se puede concluir que el proyecto de diseño de producto colaborativo multinacional es muy útil para los alumnos. Dentro de las respuestas los encuestados reconocieron que este tipo de proyecto no solo les ayudó a desarrollar competencias de trabajo en equipo y comunicación efectiva con sus socios internacionales, sino también a aprender de forma práctica sobre conceptos de diseño de productos. En general la experiencia colaborativa motivó a los alumnos a trabajar en el proyecto y estar listo para compartir información con sus socios internacionales, lo que les ayudó a terminar la asignación. Adicionalmente, a pesar que no se menciona de forma directa, se infiere que la experiencia colaborativa en sí mejoro la metodología que los estudiantes siguieron para enfrentar el problema de diseño.

Finalmente se ratificó la importancia de la comunicación cara-a-cara (video-conferencia) y del uso del correo electrónico en las interacciones con socios internacionales, ya que fueron los medios de comunicación más usados por los alumnos. Fue interesante la generalización del uso de Google Docs y drive para intercambiar datos en lugar de aplicaciones comerciales tales como Dropbox. También se validó la importancia del uso de las redes sociales para mantener una comunicación más fluida, no tan estructurada y constante entre socios de un clúster de diseño.

Debido a que uno de los problemas más grandes identificados fue la baja interacción con los socios internacionales, se sugiere fomentar más dicha colaboración. Formas en las que esto puede lograrse es asignando a los alumnos que lleven un diario de interacciones con sus socios internacionales que tenga un puntaje asociado, dando una charla sobre normas de educación para interacciones mediante TICs (netiqueta) y sugiriendo que usen las redes sociales y otras herramientas ahora disponibles para realizar conferencias de audio o video mediante dispositivos móviles de forma más sencilla y flexible tales como Skype, Line, Messenger o FaceTime.

Referente al problema de la falta de calidad de la información compartida se sugiere que los 3 entregables de colaboración internacional sean revisados y se les asigne un puntaje. Otra sugerencia para mejorar la calidad es cambiar la dinámica evaluativa del proyecto, de forma que la utilidad de la información recibida de parte de los socios internacionales sea evaluada por los grupos que conforman el clúster y tenga un puntaje asociado a la nota final de la clase.

Como trabajo futuro se sugiere continuar con la evaluación de interacciones entre los estudiantes y realizar un análisis más inferencial de la intensidad, cantidad, calidad y utilidad de la comunicación multinacional. Finalmente, se sugiere que en lugar de usar aplicaciones separadas como ANGEL, Adobe Connect Meeting y Dropbox se trabaje en generar un sistema de comunicación basado en las herramientas de Google. 


\section{Bibliografía}

Bjørn, P., \& Ngwenyama, O. (2009). Virtual team collaboration: building shared meaning, resolving breakdowns and creating translucence. Information Systems Journal, 19, 227 $-253$.

Brindley, J. E., \& Walti, C. (2009). Creating Effective Collaborative Learning Groups in an Online Environment. The International Review of Research in Open and Distance Learning, 10 (3), 1 - 18.

Bufardi, A., Xirouchakis, P., \& Duhovnik, J. E. (2005). Collaborative design aspects in the European Global Product Realization project. International Journal of Engineering Education, 950-963.

Davidow, W. H., \& Malone, M. S. (1992). The virtual corporation: Structuring and revitalizing the corporation for the 21st century (Vol. 1st edition). New York: Harper Collins Publishers.

Dekker, D. M., Rutte, C. G., \& Van den Berg, P. T. (2008). Cultural differences in the perception of critical interaction behaviors in global virtual teams. International Journal of Intercultural Relations, 32, 441 - 452.

Ebrahim, N. A., Ahmed, S., \& Taha, Z. (2009). Virtual teams: a literature review. Australian Journal of Basic and Applied Sciences, 3, 2653 - 2669.

Franzoni, C., \& Sauermann, H. (2014). Crowd science: The organization of scientific research in open collaborative projects. Research Policy, 43, 1 - 20.

Gibson, C. B., \& Cohen, S. G. (2003). Virtual teams that work. Creating Conditions for Virtual Team Effectiveness. San Francisco: Jossey-Bass.

Goodbody, J. (2005). Critical success factors for global virtual teams. Strategic Communication Management, 9, 18 - 21.

Harvey, M., Novicevic, M. M., \& Garrison, G. (2004). Challenges to staffing global virtual teams. Human Resource Management Review, 275 - 294.

Hertel, G., Geister, S., \& Konradt, U. (2005). Managing virtual teams: A review of current empirical research. Human Resource Management Review, 15, 69 - 95.

Hinds, P. \&. (2002). Distributed work. Cambridge, MA: MIT Press.

Hosseini, M. R., \& Chileshe, N. (2013). Global virtual engineering teams (GVETs): A fertile ground for research in Australian construction projects context. International Journal of Project Management, 1101 - 1117.

Karoui, M., Gürkan, A., \& Dudezert, A. (2010). Virtual Team Collaboration: A Review of Literature and Perspectives. Proceedings of the Sixteenth Americas Conference on Information Systems. Lima, Peru. 
Lee-Kelley, L., \& Sankey, T. (2008). Global virtual teams for value creation and project success: A case study. International Journal of Project Management, 26, 51 - 62.

Lee-Kelley, L., Crossman, A., \& Cannings, A. (2004). A social interaction approach to managing the "invisibles" of virtual teams. Industrial Management \& Data Systems, 104(8), 650 - 657.

May, A., \& Carter, C. (2001). A case study of virtual team working in the European automotive industry. International Journal of Industrial Ergonomics, 171-186.

Maznevski, M. L., \& Chudoba, K. M. (2000). Bridging space over time: global virtual team dynamics and effectiveness. Organization Science, 11(5), 473 - 492.

Palloff, R. M., \& Pratt, K. (2005). Collaborating online: Learning together in community. San Francisco, CA: Jossey-Bass.

Qamhiyah, A. Z., \& Ramond, B. (2005). Internationalization of the Undergraduate Engineering Program (Part 2): Application Example. International Journal of Engineering Education, 21(2), 257 - 261.

Robert, L. P., Jr., Dennis, A. R., \& Hung, Y. C. (2009). Individual swift trust and knowledgebased trust in face-to-face and virtual team members. Journal of Management Information Systems, 26(2), 241 - 279.

Sclater, N., Grierson, H., Ion, W. J., \& MacGregor, S. P. (2001). Online Collaborative Design Projects: Overcoming Barriers to Communication. International Journal of Engineering Education, 17(2), 189 - 196.

Scott, S., \& Einstein, W. (2001). Performance appraisal in team-based organizations: One size does not fit all. Academy of Management Executive, 15(2), 89 - 102.

Shachaf, P. (2008). Cultural diversity and information and communication technology impacts on global virtual teams: an exploratory study. Information and Management, 45(2), $131-142$.

Siemens, G. (8 de October de 2002). Elearnspace. Obtenido de http://www.elearnspace.org/Articles/Interaction.htm

Steward, B. L., Mickelson, S. K., \& Brumm, T. J. (2005). Continuous Engineering Course Continuous Engineering Course Multiple Assessment. International Journal of Engineering Education, 21(2), 277 - 287.

Tseng, H. W., \& Yeh, H.-T. (2013). Team members' perceptions of online teamwork learning experiences and building teamwork trust: A qualitative study. Computers \& Education, $63,1-9$.

Wang, L., Weiming, S., Xie, H., Neelamkavil, J., \& Pardasani, A. (2002). Collaborative conceptual design - state of the art and future trends. Computer Aided Design, 981-996. 
Wilczynski, V., \& Jennings, J. J. (2003). Creating Virtual Teams for Engineering Design. International Journal of Engineering Education, 19(2), 316-327.

Zavbi, R., \& Tavcar, J. (2004). Preparing undergraduate students for work in virtual product development teams. Computers \& Education, 357-376.

Zimmermann, A. (2011). Interpersonal relationships in transnational, virtual teams: towards a configurational perspective. International Journal of Management Reviews, 59 - 78.

"LA REVISTA INNOVARE NO SE HACE RESPONSABLE EN NINGÚN CASO DE LOS CONTENIDOS, DATOS, CONCLUSIONES U OPINIONES VERTIDAS EN LOS ARTÍCULOS PUBLICADOS, SIENDO ESTA RESPOSABILIDAD EXCLUSIVA DEL (DE LOS) AUTOR (AUTORES)" 